\title{
Caractérisation pathologique in vitro de deightoniella torulosa (Syd.) Ellis sur les cultivars de bananiers figue sucrée (musa AA), grande naine (musa AAA) et orishele (musa AAB)
}

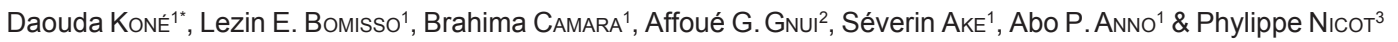 \\ ${ }^{1}$ Laboratoire de Physiologie Végétale, UFR Biosciences, Université de Cocody-Abidjan 22 BP 582 Abidjan 22, Côte-d'Ivoire \\ ${ }^{2}$ Université d'Abobo - Adjamé, UFR Sciences de la Nature, 02 BP 801 Abidjan 02, Côte-d'Ivoire \\ IINRA - Avignon Station de Pathologie Végétale, Domaine St Maurice 84143 Montfavet Cedex - France \\ *Auteur pour les correspondances (E-mail : daoukone@yahoo.fr) \\ Reçu le 03-05-2006, accepté le 01-03-2007.
}

\begin{abstract}
Résumé
Deightoniella torulosa est un champignon fréquemment associé aux attaques des cercosporioses sur les principaux cultivars de bananier en Côte-d'Ivoire. Les symptômes qu'il induit sont mal connus et les attaques occasionnées en plantation sont négligées. Le champignon a été isolé et son pouvoir pathogène a été mis en évidence sur des fragments de feuilles et des feuilles entières de différents cultivars de bananiers en utilisant différentes concentrations de spores : à $10^{4}, 5.10^{4}$ et $10^{5}$ spores $/ \mathrm{ml}$. Des coupes histologiques ont été aussi réalisées. Les résultats ont mis en évidence l'activité parasitaire du champignon sur les fragments de feuilles et sur les feuilles entières. La période d'incubation est de 3 à 5 jours. La germination des spores débute en moins d'une heure et atteint 95 à $100 \%$ au bout de 4 heures. L'évolution des symptômes varie en fonction des cultivars. L'ampleur des attaques s'est révélée importante lorsque les concentrations de spores sont élevées. Le champignon provoque des attaques sévères pour des concentrations de $10^{5}$ spores / ml. Ainsi, Deightoniella torulosa induit seul des attaques, colonise les tissus du limbe foliaire et contribue à la sénescence prématurée des feuilles de bananiers.
\end{abstract}

Mots clés : Deightoniella torulosa, activité parasitaire, inoculations, cultivars de bananiers, sénescence, Côte-d'Ivoire.

\begin{abstract}
In vitro pathogenic characterisation of deightoniella torulosa (Syd.) ellis on cultivated banana figue sucree (musa $A A)$, grande naine (musa $A A A)$ and orishele (musa $A A B$ )

Deightoniella torulosa is a fungus usually associated with symptoms of black sigatoka leaf streak in Côte-d'Ivoire. The pathogenic activity of the fungus is not well known and its effect on field is neglected. The fungus has been isolated and its pathogenic activity has been determined on leaves of cultivated banana through the use of different concentrations of conidia: $10^{4}, 5.10^{4}$ and $10^{5}$ spores / $\mathrm{ml}$. Inoculated samples have been fixed for histological observations. Results showed stomata penetration and pathogenic activity of fungus beginning from the third ( $3^{r d}$ ) to the fifth $\left(5^{\text {th }}\right)$ day after inoculation. We observed that conidia germination begins in less than 1 hour and reaches 95 to $100 \%$ within 4 hours. The progress of the disease varies according to the according to the banana cultivars and the amount of conidia. $10^{5}$ spores $/ \mathrm{ml}$ have induced symptoms. Then, Deightoniella torulosa alone could induce symptoms and was involved in rapid necrosis of infected leaves in natural conditions.
\end{abstract}

Key words : Deightoniella torulosa, pathogenic activity, inoculations, cultivated banana, Côte-d'Ivoire. 


\section{Introduction}

Les bananiers (bananier de dessert et bananier plantain) sont l'objet d'attaques très variées dues à des microorganismes de diverses natures, constituant de nos jours les contraintes majeures. Parmi ceux-ci Mycosphaerella fijiensis est l'espèce la plus redoutée en raison des pertes qu'elle occasionne (Stover, 1983; Mobambo et al., 1996b; Jones, 2000). Les espèces Mycosphaerella eumusae (Carlier et al., 2000), Cladosporium musae (Koné, 1998 ; Jones, 2000; Surridge et al., 2003 ; Sahlan et al., 2004) et Deightoniella torulosa (Jones, 2000) ont été identifiées entre autres parasites de la phyllosphère des bananiers. $D$. torulosa serait considéré comme un parasite de faiblesse rencontré sur les feuilles et les fleurs de certains cultivars de bananiers (Stover, 1972).

Les plantes dans les conditions défavorables et sous de fortes humidités sont plus sensibles aux infections. Cependant, les symptômes sont beaucoup plus importants sur Abaca que sur bananiers (Jones, 2000). Le cultivar 'Bungolanon', très largement planté en Amérique Central est affecté à $14 \%$ (Stover, 1972) par $D$. torulosa. Ces attaques sont aussi observées sur les jeunes feuilles et les feuilles âgées de Ensete en Sidamo et dans les régions Nord de Omo en Ethiopie (Jones, 2000). Les symptômes apparaissent sous la forme de petites taches nécrotiques de 1 à $2 \mathrm{~mm}$ de diamètre et évoluent en taille avec une bordure noire (Jones, 2000). Les taches matures peuvent atteindre $25 \mathrm{~mm}$ de diamètre ou plus et peuvent former par coalescence des bandes de nécroses. Les lésions peuvent prendre différentes formes et sont entourées d'un halo jaune chez Abaca. Les attaques peuvent se manifester sur le pétiole et le pseudo-tronc chez Abaca. Chez le bananier, les cultivars du sous groupe Cavendish sont attaqués (Jones, 2000).

L'agent pathogène a été décrit par Ellis (1957), Meredith (1961a, b) et Subramanian (1968). Les conidiophores du champignon sortent isolement ou en petits groupes. Ils sont bruns et présentent un renflement à leur sommet. Les conidies sortent isolement au sommet des conidiophores. Elles sont curvées, obpyriform à obclavate, subhyaline à olive avec 13 cloisons. Ces conidies ont 35-70 $\mu \mathrm{m}$ de long sur 13-25 $\mu \mathrm{m}$ de large. Pendant la germination, le tube germinatif produit des appressoria, et les feuilles sont directement attaquées à travers leur épiderme. Les spores sont produites pendant les périodes de fortes humidités et aussi avec la rosée (Meredith, 1961a). Cependant, elles ne sont pas transmises sur de longues distances et perdent leur viabilité en 4 jours sous des conditions d'humidité inférieures à $95 \%$ (Meredith, 1961c). Concernant les interactions hôte-pathogène, peu d'informations sont connues sur Deigthoniella torulosa.

En Côte d'Ivoire, Deightoniella torulosa est associé aux cercosporioses et autres maladies fongiques. Cela rend difficile la détermination de la nature des anomalies et l'estimation des dégâts qui en résultent. II s'agit d'une anomalie peu connue en bananeraie, mais qui dans certaines conditions pourrait s'avérer destructrice dans la mesure où le pathogène est fortement associé aux attaques dues à Mycosphaerella fijiensis, notamment sur le cultivar de bananier plantain Orishele. Dans les régions où les cultivars Abaca sont cultivés, il est recommandé d'utiliser les variétés résistantes lorsque la maladie cause des attaques majeures (Jones, 2000). Dans le souci de lutter contre les cercosporioses, notamment en milieu paysan pour accroître les productions et garantir la sécurité alimentaire, l'introduction de variétés tolérantes est de plus en plus envisagée. Le comportement de ces nouvelles variétés est évalué vis-à-vis des contraintes en plantation avant de les soumettre aux producteurs (N'Guessan et al., 2000). Il est donc utile d'identifier et de caractériser chaque bioagresseur, puis de déterminer son pouvoir pathogène. $D$. torulosa est un champignon qui a été pour la première fois isolé et identifié en Côte d'Ivoire à cause de sa fréquence d'association aux symptômes foliaires dus à $M$. fijiensis.

La présente étude a été envisagée en vue de caractériser in vitro le pouvoir pathogène de $D$. torulosa sur les cultivars de bananiers Orishele (Musa AAB), Grande Naine (Musa AAA) et Figue Sucrée (Musa AA) qui sont les plus fréquemment rencontrés en Côte-d'Ivoire. La connaissance des premiers signes d'infection du champignon permettra d'élucider la manifestation des symptômes extérieurs par rapports à ceux des autres espèces pathogènes que sont $C$. musae et $M$. fijiensis qui sont bien conus. Ce travail aidera à la prise en compte des attaques de $D$. torulosa 
dans l'évaluation du comportement des cultivars locaux et de nouvelles introductions de bananiers pour leur tolérance aux parasites foliaires en Côte d'ivoire.

\section{Matériel et méthodes}

\subsection{Matériel végétal et fongique}

Les inoculations ont été réalisées sur des fragments de feuilles et des feuilles entières de vivo plants âgés de 3 mois, après sevrage, sous abris. Ces plantes sont issues de cultivars Orishele (Musa AAB), Grande Naine (Musa AAA) et Figue Sucrée (Musa AA). Les cultures de Deightoniella torulosa sont obtenues par isolement monospore à partir de feuilles nécrosées du cultivar Orishele. Ces isolements sont réalisés sous un microscope en prélevant les spores une à une pour les transférer sur un milieu PDA.

\subsection{Méthodes}

\subsubsection{Préparation des suspensions de spores, inoculations et observations}

Pour évaluer la germination, $800 \mu \mathrm{l}$ de suspensions de spores calibrée à $10^{3}$ spores / $\mathrm{ml}$ ont été étalés sur PDA et V8. Le milieu PDA à l'origine est un milieu d'isolement et de croissance tandis que le milieu V8 est un milieu de sporulation. Ces deux milieux de compositions différentes ont été utilisés pour mettre en évidence la production de spores et déterminer le pouvoir pathogène des spores qui en sont issues. Les délais de germination ont été déterminés en observant et en dénombrant le nombre de conidies germées. Les suspensions conidiennes ont été préparées à partir de colonies âgées de 10 jours sur milieu de culture PDA à la température de $29 \pm 2{ }^{\circ} \mathrm{C}$. Les suspensions de spores récupérées à l'aide d'une spatule sont ajustées aux concentrations de $10^{4}, 5.10^{4}$ et $10^{5}$ spores / $\mathrm{ml}$ à l'aide d'une cellule double de numérotation de Malassez. Les inoculations ont été réalisées sur des fragments de feuilles pour déterminer la concentration de spores qui induit les symptômes avant de procéder à des inoculations sur des plantes entières. Les fragments de feuilles de $6 \mathrm{~cm}^{2}$ sont issus des premières ou deuxièmes plus jeunes feuilles des plants de bananiers âgés de 3 mois. Ces fragments sont mis en nombre de quatre sur un milieu de survie contenant $25 \mathrm{mg}$ de Benzimidazole, $20 \mathrm{~g}$ d'Agar et $20 \mathrm{~g}$ de saccharose pour $1 \mathrm{~L}$ de milieu. Les inoculations ont été faites avec des suspensions de spores calibrées à $10^{4}$, $5.10^{4}$ et $10^{5}$ spores $/ \mathrm{ml}$. Dans la même boîte de Pétri, chaque fragment est inoculé en déposant à son centre $10 \mu$ d'une des concentrations de spores à l'aide d'une micropipette. Ces fragments constituent les essais. Dans chaque boîte de Pétri, un fragment de feuille servant de témoin est inoculé avec $10 \mu \mathrm{l}$ d'eau distillée stérile. Au total 100 boîtes ont été préparées correspondant pour chaque cultivar à un total de 100 fragments inoculés par concentration et 100 fragments témoins. Les boîtes contenant les fragments inoculés sont maintenues à la température de $29 \pm 2{ }^{\circ} \mathrm{C}$ sous une photopériode de 12 heures. L'expérience a été répétée 3 fois dans le temps.

Les observations ont été effectuées tous les jours après les inoculations. Elles ont consisté pour chaque cultivar à déterminer la période d'incubation, à décrire les symptômes et à déterminer leur évolution en fonction des concentrations de spores. Les premiers symptômes microscopiques et macroscopiques ont été notés et décrits à partir des observations régulières de leur forme et de leur coloration. Le mode de pénétration du champignon est mieux observé en trempant les fragments de feuilles dans du $\mathrm{KOH}$ (4\%) pendant $24 \mathrm{~h}$. Les fragments décolorés ont été ensuite colorés au bleu coton avant de les observer au microscope photonique. L'évolution des symptômes a été comparée 10 jours après les inoculations en mesurant pour chaque cultivar la longueur et la largeur des taches.

\subsubsection{Impact de la maladie sur la dégradation de la chlorophylle}

L'impact de la maladie sur l'activité photosynthétique a été mis en évidence en déterminant la teneur en chlorophylles totales aux $7^{\text {ème }}$ et $10^{\text {ème }}$ jours après les inoculations. Cinq lots de 10 fragments ont été constitués à partir de 50 fragments inoculés et 50 témoins. Sur chaque fragment foliaire inoculé ou témoin, un disque de $1 \mathrm{~cm}$ de diamètre a été prélevé et pesé. Les pigments foliaires sont ensuite extraits par broyage dans l'acétone suivi d'un dosage au 
spectrophotomètre, aux longueurs d'ondes de $645 \mathrm{~nm}$ et $663 \mathrm{~nm}$. La teneur en chlorophylles totales a été déterminée selon la formule de $\mathrm{Mc}$ Kinney $=20,2 \mathrm{DO}_{645}+8,2 \mathrm{DO}_{663} \mathrm{mg} / \mathrm{l}$ de solution.

\subsubsection{Etudes ultrastructurales des feuilles témoins et inoculées}

Les échantillons découpés en fragments d'environ $1 \mathrm{~mm}^{2}$ ont été fixés par trempage dans un tampon contenant du cacodylate, du glutaraldehyde ( $25 \%$ dans l'eau) et de l'eau en proportion 4:1:1 (4 vol: $1 \mathrm{vol}: 1 \mathrm{vol})$. Le pH du cacodylate a été ajusté à 8 . Ce trempage a été suivi d'un dégazage pendant $10 \mathrm{~min}$ (3 fois) dans un dessiccateur à l'aide d'une pompe à vide. Le tampon a été ensuite remplacé par un nouveau tampon de même composition que le premier et les échantillons sont laissés pendant 4 heures, puis rincés trois fois pendant une heure dans le tampon cacodylate de sodium contenant $3 \%$ de glucose. Les fragments rincés trois fois ont été laissés une nuit dans le tampon de rinçage. La post fixation a lieu pendant $2 \mathrm{~h}$ dans un tampon composé du tetroxyde d'osmium et du cacodylate contenant $3 \%$ de glucose. Par la suite, cinq rinçages de $5 \mathrm{~min}$ ont été effectués à l'eau distillée. La déshydratation a été faite par plusieurs rinçages dans des bains croissants d'éthanol. Après les rinçages à l'acétone, les échantillons ont été trempés dans 2 bains de 30 min dans l'oxyde de propylène. La préimprégnation a lieu dans un mélange d'araldite + oxyde de propylène et l'imprégnation dans 4 bains de résine pure (araldite) pendant $1 \mathrm{~h} /$ bain. Les échantillons sortis du $4^{\text {ème }}$ bain ont été inclus dans la résine pure contenue dans des gélules disposées dans une plaque ELISA pendant $72 \mathrm{~h}$. Les coupes semi fines ont été réalisées à l'aide d'un microtome puis les préparations ont été contrastées avec du bleu de méthylène avant de les observer. L'épaisseur des feuilles a été mesurée avec le logiciel Biocom Visiolab T280 après l'acquisition des images à partir d'un microscope équipé d'une caméra.

\subsubsection{Inoculations sur des plantes entières}

Après avoir déterminé sur des fragments de feuilles, la concentration qui induit les symptômes, cette concentration a été utilisée pour inoculer la variété Grande Naine (Musa AAA). Les inoculations ont été effectuées sur les deux plus jeunes feuilles (cv Grande Naine) issues de bananiers âgés de 3 mois et maintenues sous abris dans les conditions d'humidité proches de la saturation et à une température entre 29 et 31 ${ }^{\circ} \mathrm{C}$. Ces inoculations ont été faites sur la face inférieure des feuilles par pulvérisation d'une concentration de spore calibrée à $10^{5}$ spores / $\mathrm{ml}$. Dix plantes ont été inoculées par concentration de spores et 10 plantes inoculées avec l'eau distillée stérile ont servi de témoin. Les plantes inoculées sont maintenues sous une cage d'inoculation dans les conditions de photopériode de $12 \mathrm{~h}$. Les inoculations ont été répétées 3 fois dans un intervalle de 3 semaines en vue de confirmer les délais d'apparition et la caractérisation des symptômes observés suite à la première inoculation. Après 24 heures, les plantes inoculées et les plantes témoins sont arrosées avec de l'eau stérile en vue de maintenir l'humidité élevée sous un abris d'inoculation. Ces arrosages sont effectués pendant 72 heures à raison de 2 arrosages par jour. Les observations sont effectuées tous les jours à partir de 24 heures après les inoculations en vue de noter les délais d'apparition des symptômes et leur évolution.

Les résultats ont été soumis à l'analyse de variance (ANOVA) avec le logiciel STATISTICA 5.0. Les moyennes ont été ensuite comparées par le test de Newman-Keuls au seuil de $5 \%$.

\section{Résultats}

\subsection{Description du champignon et étapes de l'infection}

La germination des conidies commence 30 à 60 min après leur étalement sur milieu PDA et V8 (Fig. 1). Plus de $25 \%$ des conidies ont germé après une heure. Au bout de deux heures, le taux de germination a atteint $85 \%$ sur le milieu PDA et $90 \%$ sur le milieu V8. Après 4 heures de temps, le taux de germination est resté constant et se situe à $95 \%$ sur le milieu V8 et $100 \%$ sur le milieu PDA où la totalité des spores a germé (Fig. 1). La germination des conidies commence par l'extrémité renflée ou effilée (Fig. 2 B et C) et les premières colonies sont observées à partir du $3^{\text {ème }}$ jour qui suit l'ensemencement des spores et les colonies présentent un thalle blanchâtre (Fig. 2A). Les colonies prennent une coloration noirâtre dans les parties âgées à partir 
du $5^{\text {ème }}$ jour puis la colonie entière finira par devenir brun noirâtre au 10 ème jour (Fig. 2A) avec présence de nombreuses conidies.

Lorsque les spores sont inoculées sur des fragments de feuilles, le germination a lieu dans les mêmes délais que ceux décrits précédemment. Le tube germinatif évolue avant de former à son extrémité un stomatopode (Fig. 2D). Les premiers symptômes apparaissent 48 à 72 heures après les inoculations. Ils se présentent sous l'aspect d'une petite tache de décoloration ovale, jaunâtre avec un centre brun rouille d'environ $2 \mathrm{~mm}$ de long sur $1 \mathrm{~mm}$ de large en moyenne qui va évoluer pour donner des taches ovales plus larges (Fig. 2E).

Le nombre de fragments attaqués est fonction de la pression d'inoculum et du temps qui suit l'inoculation (Tableau 1). Ainsi 4 jours après l'inoculation, les taux d'attaques vont évoluer de 20 à $60 \%$ selon les variétés pour les concentrations de spores calibrées à $10^{4}$ spores
/ ml (Tableau 1). Ces taux vont passer à plus de $80 \%$ lorsque les concentrations de spores sont de $5.10^{4} \mathrm{spores} / \mathrm{ml}$ pour atteindre $100 \%$ pour les concentrations de spores calibrées à $10^{5}$ conidies/ $\mathrm{ml}$ (Tableau 1). Aux $7^{\text {ème }}$ et $10^{\text {ème }}$ jours après les inoculations, les taux d'attaques deviennent plus importants à la concentrations de $10^{4}$ spores $/ \mathrm{ml}$ tandis que pour les quantités de spores atteignant $10^{5}$, les taux d'attaques vont atteindre $100 \%$ et demeurer constants (Tableau 1).

Les taches issues des inoculations ont des longueurs et des largeurs différentes selon les variétés (Tableau 2). Chez le cultivar Orishele, pour toutes les concentrations de spores, les taches sont plus allongées et plus larges que chez les cultivars Figue Sucrée et Grande Naine. Les différences observées entre les longueurs des taches puis entre les largeurs chez le cultivar Orishele et les cultivars Grande Naine et Figue sucrée pour chaque concentration sont significatives au seuil de $5 \%$.

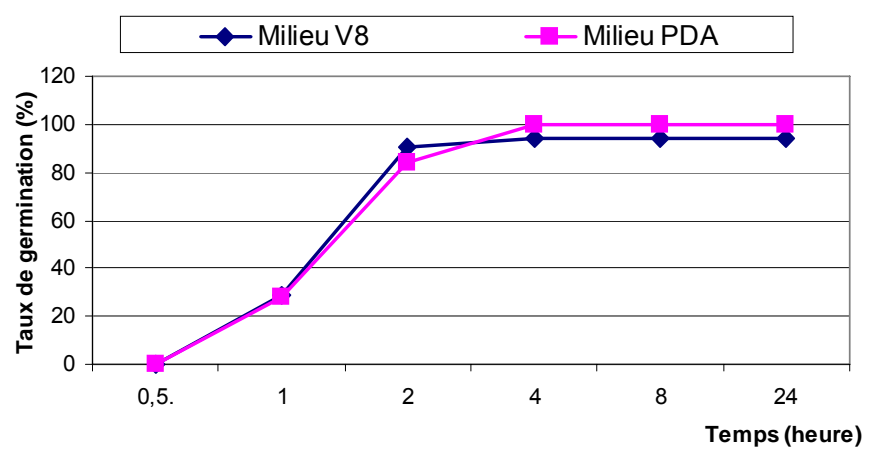

Figure 1 : Evolution de la germination des spores de Deightoniella torulosa sur les milieux de culture V8 et PDA.

Tableau 1 : Taux de fragments de feuilles de bananiers ayant exprimé des attaques de Deightoniella torulosa 4, 7 et 10 jours après les inoculations.

\begin{tabular}{lrrrrrrrrr}
\hline & \multicolumn{6}{c}{ Taux de fragments attaqués après les inoculations (\%) } \\
\cline { 2 - 9 } Cultivars & \multicolumn{3}{c}{$4 \mathrm{JAI}$} & \multicolumn{3}{c}{$7 \mathrm{JAI}$} & \multicolumn{1}{c}{$10 \mathrm{JAI}$} \\
\cline { 2 - 9 } & $10^{4}$ & $5.10^{4}$ & $10^{5}$ & $10^{4}$ & $5.10^{4}$ & $10^{5}$ & $10^{4}$ & $5.10^{4}$ & $10^{5}$ \\
\hline Orishele & 64 & 98 & 100 & 86 & 90 & 100 & 88 & 100 & 100 \\
Grande Nain & 20 & 90 & 100 & 54 & 100 & 100 & 58 & 100 & 100 \\
Figue Sucrée & 42 & 96 & 100 & 66 & 66 & 100 & 100 & 100 & 100 \\
\hline
\end{tabular}

JAl : jours après inoculations 
Tableau 2 : Dimensions des taches induites par Deightoniella torulosa en fonction de la concentration de spores inoculées sur les fragments de feuilles de 3 cultivars de bananiers.

\begin{tabular}{llccc}
\hline \multirow{2}{*}{$\begin{array}{l}\text { Dimensions des } \\
\text { taches }\end{array}$} & Cultivars & \multicolumn{3}{c}{ Concentrations de spores $/ \mathrm{ml}$} \\
\cline { 2 - 4 } Longueurs des taches & Orishele & $14,27 \pm 0,3 \mathrm{a}$ & $23,17 \pm 0,4 \mathrm{a}$ & $30,3 \pm 0,1 \mathrm{a}$ \\
$(\mathrm{mm})$ & Grande Naine & $9,07 \pm 0,2 \mathrm{~b}$ & $17,23 \pm 0,4 \mathrm{~b}$ & $18,93 \pm 0,4 \mathrm{~b}$ \\
& Figue Sucrée & $9,63 \pm 0,3 \mathrm{~b}$ & $18,93 \pm 0,4 \mathrm{~b}$ & $22,80 \pm 0,5 \mathrm{c}$ \\
\hline \multirow{2}{*}{$\begin{array}{l}\text { Largeurs des taches } \\
(\mathrm{mm})\end{array}$} & Orishele & $6,27 \pm 0,2 \mathrm{a}$ & $13,50 \pm 0,3 \mathrm{a}$ & $15,57 \pm 0,4 \mathrm{a}$ \\
& Grande Naine & $4,50 \pm 0,1 \mathrm{~b}$ & $9,00 \pm 0,1 \mathrm{~b}$ & $10,37 \pm 0,3 \mathrm{~b}$ \\
& Figue Sucrée & $5,40 \pm 0,2 \mathrm{c}$ & $10,37 \pm 0,4 \mathrm{~b}$ & $13,93 \pm 0,3 \mathrm{c}$ \\
\hline
\end{tabular}

Pour chaque paramètre (Longueur ou largeur) les comparaisons par concentrations sont faites entre les valeurs moyennes des variétés et les moyennes accompagnées des mêmes lettres ne sont pas significativement différentes.

\subsection{Désorganisation ultrastructurale liée à l'inoculation de $D$. torulosa}

Au microscopique photonique, les coupes semifines montrent une organisation normale des assises cellulaires de l'épiderme supérieur à l'épiderme inférieur (Fig. 2F) chez les feuilles témoins. Les couches épidermiques sont contiguës aux couches de cellules de l'hypoderme supérieur qui sont plus volumineuses. Après l'hypoderme supérieur, se trouve le parenchyme palissadique constitué de cellules allongées, le parenchyme lacuneux constitué de cellules arrondies avec des espaces intercellulaires. Chez les feuilles inoculées suite à la colonisation des tissus, les assises cellulaires sont désorganisées et détruites dans certaines zones (Fig. 2G) par rapport aux feuilles témoins. Le champignon est observé dans les espaces intercellulaires (Fig. $2 \mathrm{G}$ et $2 \mathrm{H}$ ). Chez les feuilles témoins, les épaisseurs qui sont d'environ 220 $\mu \mathrm{m}$ apparaissent 1,5 fois plus grandes que celles des feuilles inoculées dont les épaisseurs n'atteignent pas $125 \mu \mathrm{m}$.
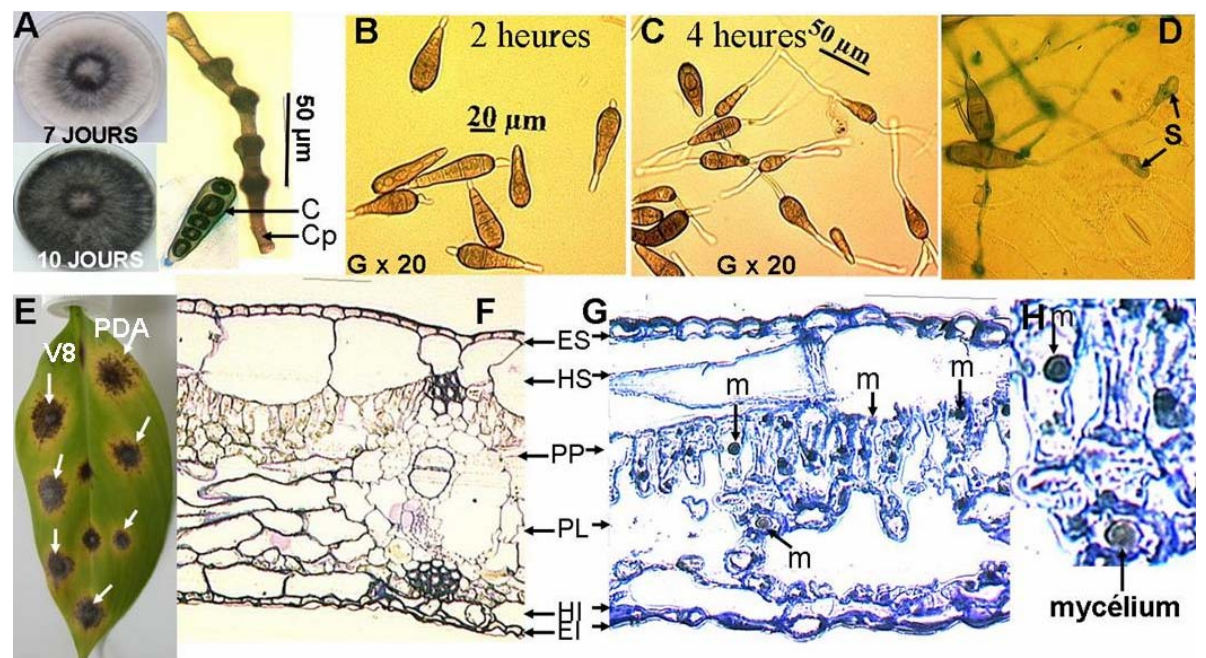

Figure 2 : Illustrations montrant les aspects culturaux de Deightoniella torulosa avec les conidies $\mathrm{C}$ et les conidiophores $\mathrm{Cp}(\mathrm{A})$, les conidies germées (B et C), la formation de stomatopodes désignés $S(D)$ précédant l'expression des symptômes après 7 jours $(E)$ puis les coupes semi fines montrant une meilleure organisation des assises cellulaires (F) par rapport aux tissus colonisés par le champignon ( $G$ et $H)$. En $F$, et $G$ : HS: hypoderme supérieur, ES: épiderme supérieur, PP: parenchyme palissadique, PL: parenchyme lacuneux, $\mathrm{HI}$ : hypoderme inférieur, El: épiderme inférieur, m: mycélium 


\subsection{Apparition et évolution des symptômes de $D$. torulosa sur les feuilles entières}

L'observation des symptômes est différente selon la dose d'inoculum. Les premiers symptômes macroscopiques apparaissent 3 à 5 jours après les inoculations (Fig. 3). Le taux de plantes contaminées est supérieur à $50 \%$ lorsque les inoculations sont effectuées avec la dose de $10^{5} \mathrm{spores} / \mathrm{ml}$. Par contre, à la dose de $510^{4} \mathrm{spores} / \mathrm{ml}$ a donné des taux d'attaque très faibles. Aucun symptôme apparent n'a été observé chez les plantes inoculées avec la dose de $10^{4}$ spores $/ \mathrm{ml}$. Les symptômes se présentent sous l'aspect de petits points de décoloration translucides qui deviennent brun rouille puis finissent par être entourés d'une marge jaunâtre plus ou moins prononcée (Fig. 3). En 7 jours, ces taches ont atteint 0,5 à $3 \mathrm{~mm}$ de long sur 0,5 à $1 \mathrm{~mm}$ de large, et sont aussi observées sur les nervures centrales et les pétioles des feuilles inoculées (Fig. $3 \mathrm{~B}$ et $\mathrm{C}$ ).
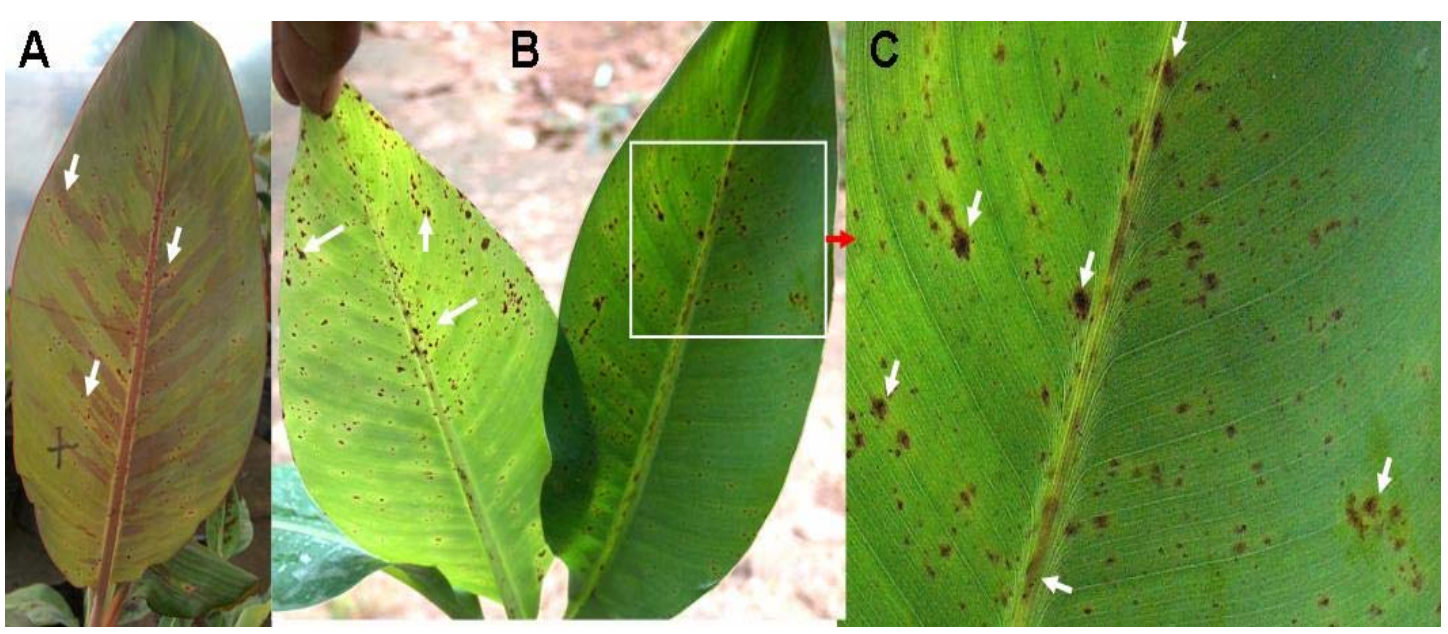

A : cultivar Orishele ; $B$ : cultivar Grande Naine ; $C$ : détail de B

Figure 3 : Aspects des symptômes de Deightoniella torulosa indiqués par des flèches blanches sur les feuilles de bananiers Orishele (Musa AAB) et Grande Naine (Musa AAA) 7 jours après l'inoculation d'une suspension conidienne calibrée à $10^{5}$ spores $/ \mathrm{ml}$.

\subsection{Impact du champignon sur la dégradation des chlorophylles}

Les attaques de $D$. torulosa se traduisent par une diminution de la teneur en chlorophylles dont les taux exprimés en pourcentage par rapport au témoin varient selon la concentration de spores (Fig. 4). Les taux de réduction sont aussi différents selon les dates d'observation (7 à 10 j) qui suivent les inoculations. Cette diminution est plus importante chez le cultivar Orishele par rapport au cultivar Figue Sucrée et Grande Naine, à toutes les concentrations de spores utilisées. Pour chaque concentration de spores, les différences observées entre les cultivars sont significatives au seuil de $5 \%$. Aux $7^{\text {ème }}$ et $10^{\text {ème }}$ jours, les différences des taux d'inhibition entre les cultivars sont plus marquées à la plus faible concentration $\left(10^{4}\right.$ spores $\left./ \mathrm{ml}\right)$ qu'aux concentrations élevées $\left(5.10^{4}\right.$ et $10^{5}$ spores $\left./ \mathrm{ml}\right)$. 


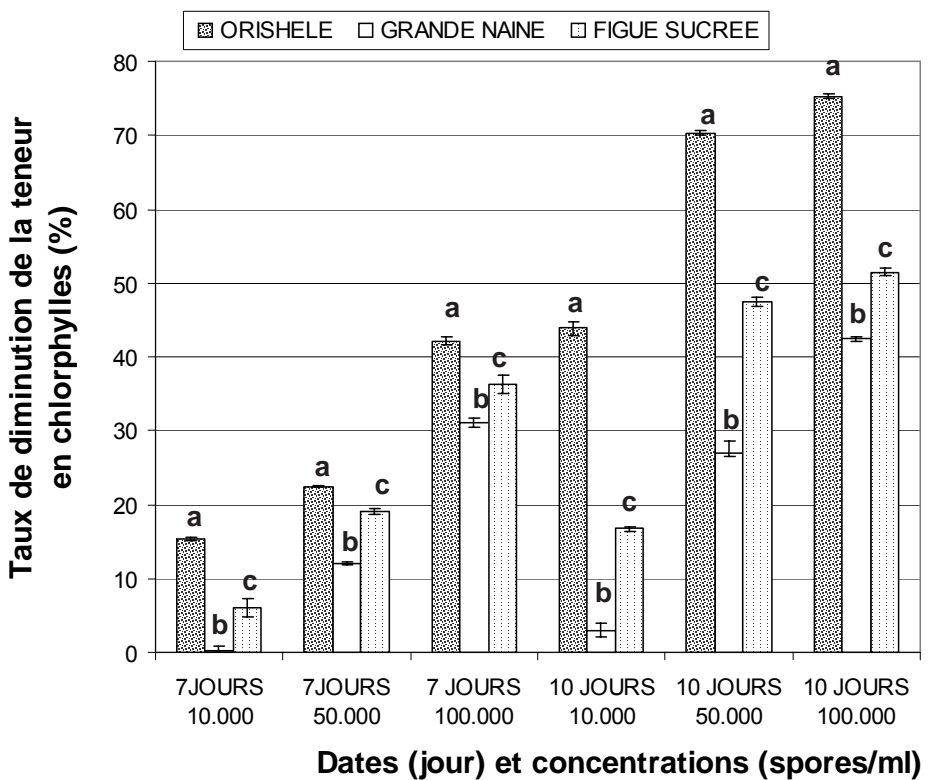

Pour chaque date en abscisse, les barres surmontées de lettres différentes indiquent les différences significatives.

Figure 4 : Taux de réduction de la teneur en chlorophylles en fonction du temps chez les cultivars Orishele, Grande Naine et Figue Sucrée inoculées avec les concentrations de spores calibrées à $10^{4}, 5.10^{4}$ et $10^{5} \mathrm{spores} / \mathrm{ml}$.

\section{Discussion}

Les cultivars de bananiers Orishele, Grande Naine et Figue Sucrée, inoculés avec les spores de Deightoniella torulosa ont exprimé des symptômes pathologiques dont l'ampleur varie selon les concentrations de spores. Les taux d'attaque varient d'un cultivar à un autre et pourrait être en rapport avec la structure génétique de chacune des variétés. En effet, les cultivars Orishele (Musa AAB) et Grande Naine (Musa AAA) sont tous des triploïdes à dominance acuminata (présence du génome $A$ ), mais se distinguent par la présence d'un gène $B$ (Musa balbisiana) chez le cultivar Orishele (Musa AAB caractérisant les bananiers plantain). Par contre, le cultivar Figue Sucré est un diploïde (Musa AA). Dans les conditions naturelles, les variétés ont montré une sensibilité différentielle vis-à-vis des attaques de parasites fongiques comme Mycosphaerella fijiensis seul (Mobambo et al., $1996 \mathrm{a}$ et b), Mycosphaerella fijiensis et Cladosporium musae (Pasberg-Gauhl \& Gauhl, 2000; Koné, 1998). C'est le cas du cultivar Figue Sucrée qui est plus sensible à la cladosporiose et moins sensible à la maladie des raies noires (Koné, 1998). Cependant, les cultivars Orishele et Grande Naine sont plus sensibles à la maladie des raies noires et très résistants à la cladosporiose (Koné et al., 2003). Par ailleurs il a été aussi démontré que la réaction des bananiers aux agressions se traduit par une production rapide des systèmes antioxydants chez les cultivars partiellement résistant, caractéristique du stress oxydatif (El Hadrami et al., 2005). Cette observation des symptômes montre que Deightoniella torulosa est virulent et qu'il peut être à l'origine de symptômes indépendamment d'un autre parasite. Les attaques sont aussi différentes selon les concentrations de spores et se manifestent par une chlorose. Le mode de pénétration est stomatique et la description des symptômes se rapproche de celle de certains auteurs (Jones, 2000). Les symptômes provoqués évoluent avec la production de métabolites toxiques qui pourrait être à l'origine de la présence du halo jaune autour des taches. En effet, chez Mycosphaerella fijiensis, la présence de halo jaune autour des taches a été attribuée à l'action des toxines liées à la 
production de métabolites toxiques par ce champignon (Natural, 1997) et chez Mycosphaerella fijiensis var. difformis (Lepoivre \& Acuna, 1997). Les essais biologiques ont montré que ces toxines induisent des nécroses (Upadhyay et al., 1997). L'activité des toxines sur le gonflement des chloroplastes a été mise en évidence chez Mycosphaerella fijiensis (Harelimana, 1997). Les dimensions des taches sont différentes selon les cultivars mais semblent évoluer lentement. Ce qui pourrait favoriser chez certains cultivars la prédominance des symptômes de la maladie des raies noires. Les inoculations sur les fragments de feuilles ont confirmé le pouvoir pathogène du champignon de même que celles effectuées sur les feuilles entières. L'absence de symptômes après l'inoculation par pulvérisation des concentrations de spores calibrées à $10^{4}$ conidies / ml montre que la pression d'inoculum pourrait influencer l'apparition et le développement des attaques. Les tissus attaqués sont envahis et colonisés par le champignon occasionnant une chlorose au niveau des zones atteintes. La teneur en chlorophylle est réduite et le taux de réduction est fonction de la pression d'inoculum. Ainsi, Deightoniella torulosa aurait contribué à la réduction des surfaces photosynthétiques et la sénescence prématurée des feuilles dans les conditions naturelles d'infestations de Mycosphaerella fijiensis avec lequel il est très fréquemment observé dans la bananeraie en Côte d'Ivoire.

\section{Conclusion}

Cette étude a permis de déterminer l'activité parasitaire de Deightoniella torulosa sur les cultivars de bananiers Orishele, Grande Naine et Figue Sucrée. Mais la sévérité du champignon exprimée par l'évolution des symptômes est différente d'un cultivar à l'autre. L'activité du champignon est aussi différente selon les concentrations de spores. Le champignon peut occasionner la dégradation de la chlorophylle et pénétrer à l'intérieur des cellules. Ce facteur pourrait être considéré dans les études épidémiologiques visant à évaluer la réaction de différents cultivars vis-à-vis du pathogène. Les concentrations de spores de $10^{5}$ spores $/ \mathrm{ml}$ sont inductrices de symptômes chez les trois variétés étudiées. Ce travail qui montre que Deightoniella torulosa induit des symptômes, ouvre les perspectives de recherches sur l'épidémiologie, les relations hôtes-pathogènes, et la recherche de méthodes de lutte ainsi que la caractérisation moléculaire des souches isolées dans différentes zones écologiques.

\section{Références citées}

Carlier J., Zapater M.-F., Lapeyre F, Jones D.R., \& Mourichon X., 2000. Septoria leaf Spot of Banana: A newly Discovered Disease Caused by Mycosphaerella eumusae (anamorph Septoria eumusae). Phytopathology 90: 884-890.

El Hadrami A., Kone D. \& Lepoivre P., 2005. Effect of juglone on active oxygen species and antioxidant enzymes in susceptible and partially resistant banana cultivars to Black Leaf Streak Disease. Eur. J. of Plant Pathol. 113 : 241-254.

Ellis M. B., 1957. Some species of Deightoniella. Mycol. Pap. 66: 12.

Fouré E., 1985. Les cercosporioses des bananiers et leurs traitements. Comportement des variétés. Etude de la sensibilité des bananiers et plantains à Mycosphaerella fijiensis MORELET au Gabon (maladie des raies noires) (suite III). Fruits 40: 393-399.

Harelimana G., Lepoivre P. Jijakli, H.\& Mourichon, X., 1997. Use of Mycosphaerella fijiensis toxins for the selection of banana cultivars resistant to Black Leaf Streak. Euphytica 96: 125-128.

Jones D.R., 2000. Diseases of banana, abaca and ensete. New York CABI press USA.

Koné D., 1998. Contribution à l'étude des cercosporioses et à la cladosporiose des bananiers en Côte d'Ivoire. Thèse de Doctorat $3^{\text {ème }}$ cycle. Laboratoire de Physiologie Végétale, UFR Biosciences, Université de Cocody-Abidjan, 218 pp.

Koné D., Aké S., Kobenan K. \& Anno A. P., 2004. Etude épidémiologique comparée de la cercosporiose noire et de la cladosporiose des bananiers sur trois cultivars triploïdes en Côte d'Ivoire. BIOTERRE 4 (4) :pp. $75-83$.

Lepoivre P. \& Acuna Ch. P., 1989. Production of toxins by Mycosphaerella fijiensis var. difformis and induction of antimicrobial compounds in banana: their relevance in breeding for resistance to black sigatoka. In: Fullerton R.A. and Stover R. $H$. (eds.) Sigatoka leaf spot diseases of banana. Proceedings of an International Workshop, March 28-April 1, 1989, San José, Costa Rica, 201-207. 
Meredith D. S., 1961a. Fruit spot ('speckle') of Jamaican bananas caused by Deightoniella torulosa (Sd.) Ellis. I-III. Transac. British Mycol. Soc. 44: 95-104.

Meredith D. S., 1961b. Spore discharge in Deightoniella torulosa (Sd.) Ellis. Ann. Bot. 25: 271-278.

Meredith D. S., 1961c. Fruit spot ('speckle') of Jamaican bananas caused by Deightoniella torulosa (Sd.) Ellis. IV. Further observations on spore dispersal. Annals of Applied Biology 49: 488-496.

Mobambo K. N., Gauhl F., Pasberg-Gauhl C. \& Zuofa K., 1996a. Season and Plant age affect evaluation of plantain for response to black Sigatoka disease. Crop Prot. 15: 609-614.

Mobambo K. N. ; Gauhl, F., Swennen, R. \& Pasberg-Gauhl, C., 1996b. Assessment of the cropping cycle effects on black leaf streak severity and yield decline of plantain hybrids. International Journal of Plant Pest Management 42: 1-8.

Natural M. P., 1989. An update on the development of an in vitro screening procedure for resistance to Sigatoka leaf spot diseases of banana. In: Fullerton R.A. and Stover R. H. (eds.) Sigatoka leaf spot diseases of banana. Proceedings of an International Workshop, March 28-April 1, 1989, San José, Costa Rica, 201-207

N'Guessan A. E. B., Koné D. , Kobenan K., Aké S. \& Tenkouano A., 2000. Caractéristiques et évaluation de comportement de quelques hybrides tétraploïdes de bananiers plantain dans le sud de la Côte - d'Ivoire. BIOTERRE, Vol 1, n'1: $6-21$.

Pasberg-Gauhl C. \& Gauhl F., 2000. Response of East African highland bananas to black leaf streak sigatoka and Cladosporium leaf speckle under tropical humid forest lowand conditions in West Africa. Acta Hort. ISHS 540: 325-334.

Sahlan M.A., Zainal A., Sariah M. \& Gurmit S., 2004. Identification of the fungus causing leaf speckle in Malaysia. In: International congress of Musa, Harnessing research to improve livelihoods, 6-9 July 2004, Penang-Malaysia P50, p. 168.

Stover R. H., 1972. Banana, Plantain and Abaca diseases. Commonwealth Mycological Institute, Kew, Survey, UK, 316 pp.

Stover R. H., 1983. Effet du Cercospora noir sur les plantains en Amérique centrale Fruits 38: 326-329.

Subramanian C. V., 1968. Deightoniella toulosa. CMI Descriptions of Pathogenc Fungi and Bacteria 165: 1-2.

Surridge A. K. J. \& Wehner F.C., Crous P.W. \& Viljoen, A., 2003. First report of Cladosporium musae on banana in South Africa. Aust. Plant Pathol. Soc. 32: 499-503.

Upadhyay R., Strobel G. A. \& Coval S., 1989. Some toxins of Mycosphaerella fijiensis. In: Fullerton R.A. and Stover R. H. (eds.) Sigatoka leaf spot diseases of banana. Proceedings of an International Workshop, March 28-April 1, 1989, San José, Costa Rica, 231-236. 